\title{
Design of auto control interface circuit for thick film heater gas sensor
}

\begin{abstract}
In this paper an automated control system of thick film resistive heater for gas sensor applications has been designed using low cost technologies. To control the desired temperature of heater, a programmable voltage with 12 bits resolution applies which can be adjusted to the appropriate temperature. The circuit consists of ADC, DAC, microcontroller, and current buffer. This circuit compensates dropt voltage across the heater which has been resulted from the ambient temperature changes. In particular, using one microcontroller for entire heater process is for the first time introduced to drive the heater, control heater temperature, and compensate ambient temperature of heater all together automatically. Fabrication of this circuit using commercially available technologies and simplicity of the circuit make it a novel and simple design in gas sensor applications. This circuit has the ability to connect to alarms and computers in order to monitoring purposes.
\end{abstract}

Keyword: Auto calibration; Thick film resistive; Control heater; Ambient temperature; Compensating temperature 